\title{
Facteurs qui influent sur le consentement au don d'organes : étude rétrospective basée dans la population
}

\author{
Jeffrey M. Singh MD MSc, Ian M. Ball MD MSc, Michael Hartwick MD Med, Eli Malus MD MSc, Karim Soliman MD, \\ John G. Boyd MD PhD, Sonny Dhanani MD, Andrew Healey MD
}

Citation : CMAJ 2021 November 15;193:E1725-32. doi : 10.1503/cmaj.210836-f

Voir la version anglaise de l'article ici : www.cmaj.ca/lookup/doi/10.1503/cmaj.210836; voir l'article connexe en anglais au www.cmaj.ca/lookup/doi/10.1503/cmaj.190761

\section{Résumé}

Contexte : Améliorer l'approche des donneurs d'organes potentiels et obtenir leur consentement pourrait favoriser l'autonomie des patients et donner accès à un plus grand nombre d'organes à transplanter. Nous avons voulu identifier les facteurs modifiables qui influent sur le consentement au don d'organes.

Méthodes : Nous avons procédé à une étude de cohorte rétrospective regroupant les adultes ( $\geq 18$ ans) consécutivement orientés vers le système de don d'organes en Ontario entre avril 2013 et juin 2019. Nous avons analysé les données cliniques et démographiques des patients, les données relatives à leurs mandataires et les particularités des approches pour le consentement au don. Les paramètres de l'étude étaient le consentement au don d'organes et le taux d'approches. Nous avons analysé les liens indépendants entre le consentement et les facteurs propres aux approches et au système.

Résultats : Nous avons identifié 34837 signalements de donneurs d'organes potentiels, dont 6548 (18,8\%) ont fait l'objet d'approches auprès de leurs mandataires en vue d'un consentement. Parmi ces derniers, 3927 (60,0\% des approches) ont mené à un consentement au don d'organes et 1883 patients $(48,0 \%$ des consentements) ont effectivement été donneurs. La raison la plus courante pour laquelle des mandataires n'ont pas été approchés en vue du consentement à un don potentiel était un retard de signalement de la part de l'équipe soignante $(45,2 \%)$. Les facteurs modifiables indépendants associés au consentement incluaient : approche téléphonique (rapport des cotes [RC] ajusté 0,46 , intervalle de confiance IC à $95 \% 0,35-0,58)$ et approche en collaboration avec le médecin et la coordination des dons (RC ajusté 1,26, IC à 95\% 1,01-1,59).

Interprétation : Le consentement au don d'organes a été associé à plusieurs facteurs modifiables. Les organisations devraient cibler des interventions visant à assurer un signalement rapide aux organisations de don d'organes, favoriser les approches en personne et promouvoir la participation des médecins au processus d'approche.
$\mathbf{F}$

aute d'organes à transplanter, beaucoup de gens meurent alors qu'ils sont en attente d'une transplantation, car la demande excède de loin l'offre. Près de 4500 personnes sont ainsi en attente d'une transplantation d'organe au Canada. Même si partout au pays, la population approuve le don d'organes ${ }^{1}$, le taux effectif de dons varie de 8,8 à 21,2 par million d'habitants's, et on n'arrive pas à créer une réserve substantielle de donneurs potentiels ${ }^{2,3}$. L'identification, le signalement et l'approche des donneurs potentiels peuvent être facilités par des politiques, des lois et des pratiques optimales ${ }^{3,4}$, même si l'efficacité des interventions varie d'une région à l'autre ${ }^{5,6}$. Certaines interventions générales ayant pour but d'accroître le nombre de donneurs n'ont pas permis de modifier les taux de consentement ${ }^{7}$, ce qui laisse supposer que l'approche au consentement gagnerait à être améliorée.

Les mandataires jouent un rôle important dans tout le processus du don d'organes, même dans les régions dotées de registres de consentement au don d'organes ou de systèmes de consentement présumé (ou opt-out, où la personne doit signifier son refus). On demande presque toujours aux mandataires l'autorisation de prélever les organes, même si un consentement au don a déjà été enregistré ${ }^{8}$ et leur taux de consentement varie 
beaucoup 9 . Les mandataires qui font face à de telles décisions s'exécutent souvent dans un contexte très chargé émotionnellement et beaucoup ignorent les volontés du patient à cet égard ${ }^{10}$. Dans ce contexte, le processus d'obtention du consentement et le soutien offert peuvent avoir un effet substantiel sur la décision. Certaines pratiques qui peuvent améliorer les taux de consentement des mandataires ont été identifiées ${ }^{11}$ et elles sont appliquées habituellement par les grandes organisations de don d'organes performantes. Plusieurs études épidémiologiques ont identifié des facteurs non modifiables associés au consentement au don d'organes (p. ex., âge, race, statut socio-économique et niveau d'instruction $)^{12-15}$. La variabilité persistante des taux de consentement laisse supposer que d'autres facteurs modifiables sont susceptibles d'influer sur la décision des mandataires vis-à-vis du consentement.

Nous avons voulu identifier les facteurs modifiables propres aux approches et aux systèmes qui influent sur le consentement au don d'organes en Ontario, au Canada.

\section{Méthodes}

\section{Conception de l'étude, situation et contexte}

Nous avons procédé à une étude de cohorte rétrospective regroupant tous les donneurs d'organes potentiels en Ontario, au Canada, qui ont été orientés vers le Réseau Trillium pour le don de vie, seule organisation à coordonner les dons d'organes dans la province. Tous les hôpitaux doivent informer le Réseau Trillium pour le don de vie lorsqu'un patient décède ou que, de l'avis d'un médecin, la mort est imminente ${ }^{16}$. Les signalements sont soumis à un triage centralisé et en cas de don potentiel, un coordonnateur dûment formé approche le mandataire au sujet du don d'organes. Depuis 2014, on a aussi affecté aux hôpitaux un médecin qui est responsable des dons d'organes sur les plans clinique et opérationnel. L'Ontario dispose d'un registre de consentement à participation volontaire en ligne dans lequel plus de $30 \%$ de la population a enregistré son consentement au don d'organes et de tissus; malgré cela, on demande encore chaque fois le consentement des mandataires. Le Réseau Trillium pour le don de vie applique d'emblée les pratiques optimales existantes (p. ex., discussions distinctes sur le pronostic et le consentement, approche par un coordonnateur dûment formé dans un environnement calme) $)^{11,17}$.

\section{Participants et sources des données}

Nous avons identifié les patients de 18 ans ou plus qui ont été orientés consécutivement vers le Réseau Trillium pour le don de vie entre le $1^{\text {er }}$ avril 2013 et le 31 juillet 2019 en interrogeant une base de données cliniques électronique (Transplant Connect). Cette cohorte inclut pour ainsi dire tous les décès de patients ventilés mécaniquement des unités de soins intensifs en Ontario parce que la conformité avérée aux critères obligatoires de signalement des patients est toujours de $95 \%$ ou plus. Tous les donneurs de notre étude avaient été ventilés mécaniquement. Des coordonnateurs dûment formés pour l'approche au don d'organes et de tissus ont consigné dans la base de données les renseignements démographiques et cliniques de chaque cas. La base de données est vérifiée régulièrement et elle est maintenue de manière à ce que les données recueillies soient complètes et de qualité pour la gestion des cas, les rapports publics et la conformité réglementaire.

\section{Expositions et paramètres d'intérêt}

Nous avons relié le code postal de la résidence de chaque patient aux données du Recensement canadien à l'aide du fichier de conversion des codes postaux de Statistique Canada ${ }^{18}$. Pour chaque patient, nous avons obtenu le quintile standardisé de revenu du ménage, la région sanitaire, le tercile d'immigration local (selon la proportion d'immigrants et de résidents non permanents) et la classification rurale ou urbaine du lieu de résidence. Pour les patients dont les mandataires ont été approchés en vue d'un consentement, nous avons obtenu les données sur les approches et les renseignements démographiques des mandataires. Nous avons classifié la religion autodéclarée des mandataires selon les grands groupes utilisés par l'Enquête nationale canadienne auprès des ménages de $2011^{19}$. Nous avons défini la « prémention » d'un don comme toute discussion consignée au dossier relativement au don d'organes initiée par l'équipe soignante avant l'approche au consentement par le coordonnateur dûment formé à cet effet; nous n'avons pas inclus les présentations ou les contacts avec le coordonnateur au don d'organes. Nous avons défini une " approche collaborative » comme une approche au consentement au cours de laquelle le médecin et le coordonnateur ont abordé les mandataires ensemble et le médecin était présent durant la discussion.

Le paramètre principal de l'étude était le consentement au don d'organes. Le paramètre secondaire était l'approche par un coordonnateur du Réseau Trillium pour le don de vie.

\section{Analyse statistique}

Nous avons généré des statistiques descriptives incluant les sousgroupes de cas approchés et de cas ayant mené à un consentement, définis a priori. Nous avons aussi analysé les cas pour lesquels le consentement a été rescindé et les cas qui n'ont pas été approchés en vue d'un don d'organes. Nous avons rapporté les données sous forme de moyennes, de médianes et de proportions, et effectué des comparaisons au moyen des tests de Student (ou test $t$ ), exact de Fisher ou du $\chi^{2}$, selon le cas. Nous avons évalué les tendances linéaires au moyen du test de tendance de Cochran-Armitage.

Nous avons évalué les liens indépendants entre les variables propres aux cas et aux consentements au moyen d'une analyse de régression logistique multivariée. Les covariables potentielles ayant des liens plausibles avec le consentement ont été dégagées par les experts du don d'organes du Réseau Trillium pour le don de vie. Nous avons remplacé les codes postaux manquants par ceux de l'hôpital de référence pour maintenir le lien avec le centre de référence d'un patient et une région géographique plus vaste à l'intérieur du système de santé hautement sectorisé de l'Ontario. Toutes les autres variables manquantes ont été traitées par imputations multiples, avec estimation finale des paramètres regroupés pour 50 ensembles comptant des données manquantes imputées. Nous n'avons pas utilisé la sélection automatisée des variables dans le modèle final.

Toutes les analyses ont été effectuées à l'aide de la version 9.4 du logiciel statistique SAS (SAS Institute). 


\section{Approbation éthique}

Le protocole de l'étude a été approuvé par le Comité d'éthique de la recherche en sciences de la santé de l'Université de Toronto (\#00034755).

\section{Résultats}

Nous avons recensé 34837 adultes signalés au Réseau Trillium pour le don de vie entre le $1^{\text {er }}$ avril 2013 et le 31 juillet 2019 (figure 1). Après triage et analyse des cas, les mandataires de 6548 patients (18,8\% de tous les signalements) ont été approchés en vue d'un consentement. Le consentement a été obtenu pour 3927 donneurs potentiels ( $60,0 \%$ des cas approchés) et 1883 ( $48,0 \%$ des cas ayant consenti) ont fait don d'au moins 1 organe, et parmi ces derniers, 1311 (69,6\% de tous les donneurs) après détermination de la mort neurologique et 572 (30,4\% de tous les donneurs) après détermination du décès cardiocirculatoire (DCC). Les dons consécutifs aux DCC provenaient de 849 donneurs potentiels ayant consenti et chez qui on a cessé les mesures de maintien des fonctions vitales (don d'organe après $D C C, 67,4 \%$ ). Les caractéristiques démographiques des patients dont les mandataires ont été approchés en vue d'un consentement au don d'organes sont présentées au tableau 1, stratifiées selon le statut à l'égard du consentement.

\section{Signalement, approche et caractéristiques du consentement}

Le nombre de cas orientés vers le Réseau Trillium pour le don de vie est passé en moyenne de 300 par mois en 2013 à 600 par mois en 2019 ( $p<0,01$ pour la tendance linéaire). La proportion de ces cas approchés en vue d'un consentement a légèrement diminué au cours de la période de l'étude d'une moyenne de $21,4 \%$ en 2013 à $17,5 \%$ en 2019 ( $p<0,001$ pour la tendance). Parmi les 34837 cas triés durant la période de l'étude, 28289 $(81,2 \%)$ n'ont pas été approchés en vue d'un don. Le plus souvent, les cas écartés avant même l'approche étaient rejetés pour des raisons médicales ( $n=14459,51,1 \%$ ) ou parce qu'il était impossible de maintenir les paramètres physiologiques ( $n=4467,15,8 \%$ ) (figure 1). En ce qui concerne les donneurs potentiels $(n=1750)$, la raison la plus fréquente pour laquelle ils n'ont pas été approchés était un retard de signalement par l'équipe soignante; $812(46,4 \%)$ de ces cas ont fait l'objet d'un signalement pendant ou après l'arrêt des mesures de maintien des fonctions vitales, plus rarement, le mandataire a refusé de parler au coordonnateur du Réseau Trillium pour le don de vie ( $n=475,1,7 \%)$, ou aucun mandataire n'était disponible ( $n=127$, $0,5 \%)$. Cela peut être considéré comme un refus, et le taux global de consentements serait alors de 55,9\% (3927 consentements au don sur 7023 cas). Les coordonnateurs n'ont pas recueilli les données démographiques complètes pour ces cas; nous ne les avons donc pas inclus parmi les refus dans notre analyse ajustée en raison de la forte proportion de données manquantes.

Des approches téléphoniques ont été tentées dans 347 cas (5,3\% des approches). Les approches téléphoniques n'ont pas été associées à la journée de la semaine, mais ont été associées à des critères géographiques $(p<0,01)$. Le consentement téléphonique a été plus fréquent dans la région sanitaire administrative du Nord-Est de l'Ontario (9,9\% de toutes les approches), principalement rurale, et il a également été plus fréquent dans les

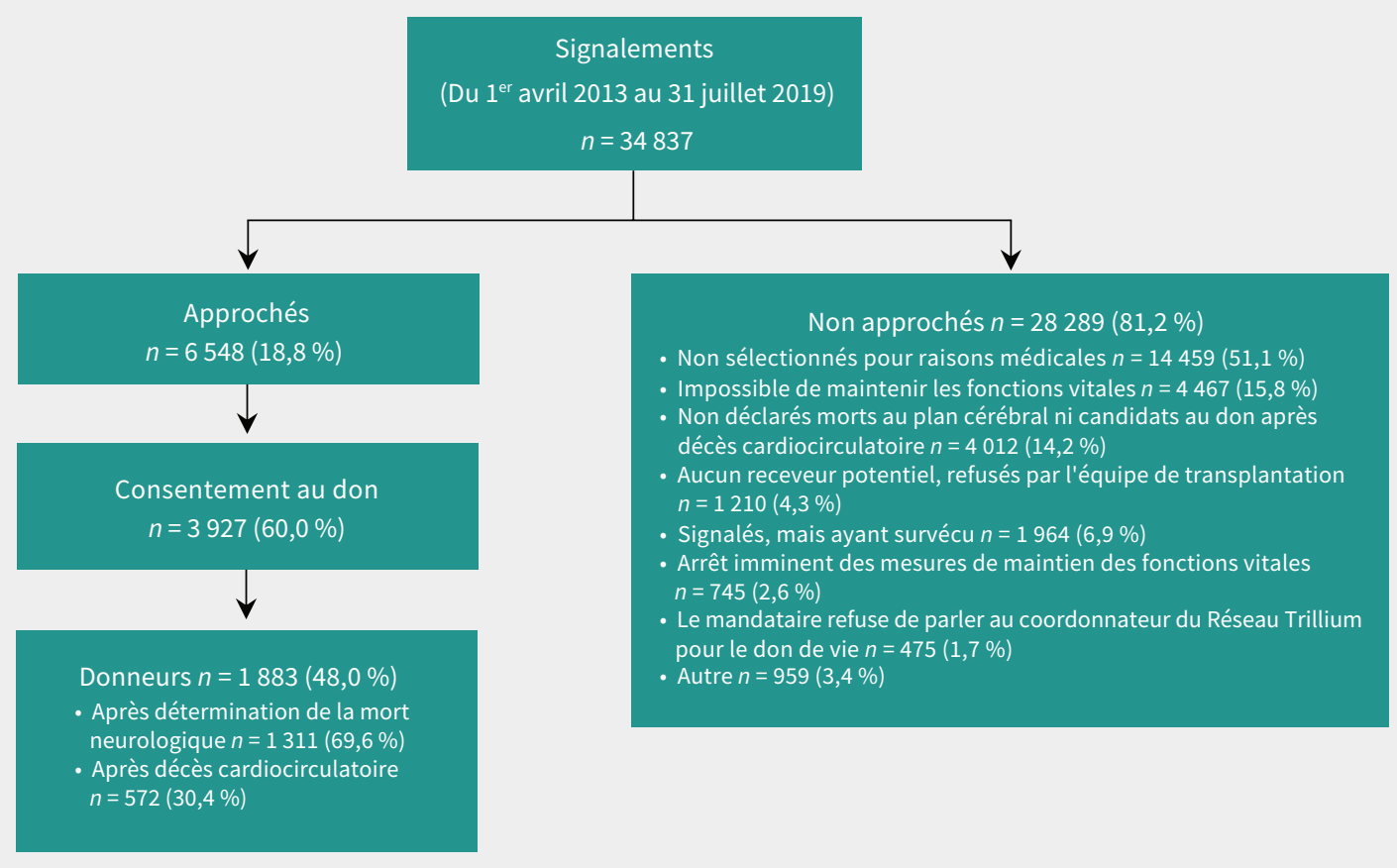

Figure 1 : Diagramme illustrant la sélection de la cohorte. 
Tableau 1 (partie 1 de 2) : Caractéristiques des patients dont les mandataires ont été approchés en vue d'un consentement, selon le statut du consentement

$N^{\text {bre }}(\%)$ de patients approchés en vue d'un don d'organes*

\begin{tabular}{|c|c|c|}
\hline Variables & $\begin{array}{c}\text { Consentement } \\
n=3927\end{array}$ & $\begin{array}{c}\text { Refus } \\
n=2621\end{array}$ \\
\hline Âge moyen, ans ( \pm É.T.) & $54,3 \pm 15,3$ & $58,5 \pm 14,0$ \\
\hline Sexe, femmes & $1553(39,6)$ & $1094(41,8)$ \\
\hline \multicolumn{3}{|l|}{ Diagnostic principal ou cause du décès } \\
\hline AVC ou autre cause neurologique & $1352(38,7)$ & $519(22,5)$ \\
\hline Arrêt cardiaque ou anoxie cérébrale & $1249(32,3)$ & $391(15,3)$ \\
\hline Traumatisme crânien ou autre & $450(13,7)$ & $94(7,8)$ \\
\hline Autre cause médicale & $361(9,1)$ & $214(15,9)$ \\
\hline Manquant & $254(10,7)$ & $831(50,6)$ \\
\hline Approche téléphonique & $126(3,2)$ & $221(8,4)$ \\
\hline Intervalle médian entre l'admission et le signalement, j (EIQ) & $1(0-4)$ & $1(0-5)$ \\
\hline \multicolumn{3}{|l|}{ Région sanitaire $†$} \\
\hline Centre & $192(5,8)$ & $211(9,4)$ \\
\hline Centre-Est & $227(6,9)$ & $177(7,9)$ \\
\hline Centre-Ouest & $218(6,6)$ & $1465(6,4)$ \\
\hline Champlain & $379(11,4)$ & $195(8,6)$ \\
\hline Érié St. Clair & $216(6,5)$ & $135(6,0)$ \\
\hline Hamilton Niagara Haldimand Brant & $351(10,6)$ & $170(7,5)$ \\
\hline Mississauga Halton & $194(5,9)$ & $165(7,3)$ \\
\hline Nord-Est & $179(5,4)$ & $153(6,8)$ \\
\hline North Simcoe Muskoka & $63(1,9)$ & $55(2,4)$ \\
\hline Nord-Ouest & $90(2,7)$ & $89(4,0)$ \\
\hline Sud-Est & $187(5,7)$ & $136(6,0)$ \\
\hline Sud-Ouest & $269(8,1)$ & $191(8,5)$ \\
\hline Toronto Central & $592(17,9)$ & $349(15,5)$ \\
\hline Waterloo Wellington & $155(4,7)$ & $85(3,8)$ \\
\hline
\end{tabular}

petites agglomérations urbaines (p. ex., 7,7\% pour la région sanitaire Hamilton Niagara Haldimand Brant et $7,1 \%$ pour celle de Waterloo Wellington) et les banlieues entourant le plus grand centre urbain $(6,6 \%)$.

Le nombre de signalements pour lesquels le consentement au don d'organes a été obtenu a augmenté constamment durant la période de l'étude, passant de 36 consentements par mois en 2013 à 64 consentements par mois en 2019 ( $p<0,01$ pour la tendance). Toutefois, la proportion de consentements par rapport au nombre d'approches n'a pas changé avec le temps (données non illustrées).

\section{Rôle de l'équipe soignante dans la prémention et l'approche}

La plupart des approches pour le consentement au don d'organes auprès des mandataires ont été réalisées par le coordonnateur seul. Lorsque le médecin du patient était présent avec le coordonnateur pour l'approche ( $n=441,6,7 \%)$, le taux de consentement était plus élevé ( $n=310,70,3 \%$ ) qu'avec le coordonnateur seul
( $n=3617,59,2 \% ; p<0,01$ ). Une prémention du don d'organes par un professionnel de la santé avant l'approche du coordonnateur dûment formé a été consignée dans 2252 cas (34,4\%); 1651 (73,3\%) ont par la suite été approchés par le coordonnateur et, parmi ceux-ci, 891 (54,0\%) ont consenti au don. Dans les cas où le mandataire a commencé par refuser le don après la prémention ( $n=876,38,9 \%), 457$ (52,2\%) ont été approchés de nouveau par un coordonnateur dûment formé, et cela a permis d'obtenir 42 consentements $(9,2 \%)$ de plus. Lorsqu'après la prémention le mandataire avait autorisé le don ou était indécis ( $n=954,42,4 \%$ ), 842 (88,2\%) ont été approchés de nouveau par un coordonnateur dûment formé et 661 (78,5\% des secondes approches) ont donné leur consentement. Étant donné que chaque cas admissible ayant donné une réponse non négative lors d'une prémention par le médecin a été approché ensuite par un coordonnateur, nous avons aussi été incapables de déterminer l'incidence indépendante de la prémention par le médecin ou de procéder à une analyse multivariée exploratoire. 
Tableau 1 (partie 2 de 2) : Caractéristiques des patients dont les mandataires ont été approchés en vue d'un consentement,

selon le statut du consentement

$\mathrm{N}^{\text {bre }}(\%)$ de patients approchés en vue d'un don d'organes*

Variables

Taille de la population

Grand centre urbain ( $\geq 100000$ personnes)

Centre moyen (de 30000 à 99999 personnes)

Petit centre (de 1000 à 29999 personnes)

Région rurale

Quintile de revenu des ménages

Quintile de revenu supérieur

Quintile de revenu moyen-élevé

Quintile de revenu moyen

Quintile de revenu moyen-faible

Quintile de revenu inférieur

Tercile d'immigration

Tercile inférieur de la population d'origine étrangère

Tercile moyen de la population d'origine étrangère

Tercile élevé de la population d'origine étrangère

Religion du mandataire

Catholique ou autre religion chrétienne

Non précisée

Agnostique, athée ou areligieux

Musulmane

Bouddhiste

Hindoue

Spiritualité autochtone

Chrétienne orthodoxe

Juive

Mandataire non de langue anglaise

Médecin chargé des dons à l'hôpital de référence

Prémention par l'équipe soignante

Remarque $: \mathrm{EIQ}=$ écart interquartile, É.T. = écart type.

*Sauf indication contraire.

tRéseaux locaux d'intégration des services de santé en Ontario (RLISS).

Facteurs indépendants qui influent sur le consentement Avant l'imputation multiple, les données de 5020 cas ( $76,7 \%$ de tous les dossiers) étaient complètes. Aucune variable ne présentait plus de $5 \%$ de données manquantes, sauf en ce qui concerne la religion du mandataire, pour laquelle les données manquantes s'élevaient à plus de $10 \%$. Après l'imputation multiple, notre analyse multivariée a mis au jour plusieurs facteurs indépendants propres au patient, aux approches et aux caractéristiques démographiques qui influent sur le consentement au don d'organes (tableau 2).

L'avancement en âge du patient (rapport des cotes [RC] 0,99, intervalle de confiance [IC] à 95\% 0,98-0,99 par tranche de 10 ans) et l'approche téléphonique pour le consentement (RC 0,46, IC à

Consentement
$n=3927$$\quad \begin{gathered}\text { Refus } \\ n=2621\end{gathered}$

Valeur $p$
$2872(74,2)$

$347(9,0)$

$222(5,7)$

$432(11,2)$

$937(24,9)$

$865(23,0)$

$798(21,2)$

$408(10,8)$

$759(20,2)$

$2165(58,6)$

$1087(29,4)$

$443(12,0)$

$1453(57,6)$

$500(15,9)$

$412(16,3)$

$26(1,0)$

$35(1,4)$

$44(1,7)$

$34(1,4)$

$17(0,7)$

$15(0.6)$

$1873(47,7)$

$3141(80,0)$

$2033(51,8)$
$<0,001$

$1920(75,1)$

$215(8,4)$

$79(3,1)$

$343(13,4)$

0,6

$657(26,4)$

$555(22,3)$

$499(20,1)$

278(11,2)

$496(20,0)$

$<0,001$

$1347(56,1)$

$679(28,3)$

$374(15,6)$

$<0,001$

$613(42,8)$

$291(20,3)$

$106(7,4)$

$77(5,4)$

$47(3,3)$

$35(2,4)$

$54(3,8)$

$27(1,9)$

$18(1,3)$

$1182(45,1)$

0,04

$2062(78,7)$

0,2

$998(38,1)$
$95 \%$ 0,35-0,58) ont été négativement associés au consentement. Les mandataires se disant de spiritualité autochtone ou bouddhiste, orthodoxe chrétienne, hindoue, juive ou musulmane avaient une probabilité significativement moindre de consentement que ceux qui se disaient chrétiens, et ceux qui se disaient athées, agnostiques ou areligieux avaient une probabilité significativement plus élevée de consentement (tableau 2). Le consentement a présenté une variabilité interrégionale significative, 4 régions sanitaires ont affiché une probabilité ajustée de consentement statistiquement différente de celle de la région de base, Toronto Central (tableau 2). L'approche collaborative a indépendamment été associée au consentement (RC 1,26, IC à 95\% 1,01-1,59). 
Tableau 2 : Analyse multivariée ajustée pour tenir compte du consentement au don d'organes

\section{RC (IC à $95 \%)$ pour le consentement}

\section{Variables}

Âge, ans (par tranche de 10 ans)

Sexe, hommes (c. femmes)

Approche téléphonique

Médecin chargé des dons à l'hôpital de référence

Intervalle entre l'admission et le signalement (par tranche de $7 \mathrm{j}$ )

Prémention par l'équipe soignante

Approche collaborative au consentement avec le médecin

Taille de la population (référence : grand centre urbain, population $\geq 100000$ )

Centre moyen (de 30000 à 99 999)

Petit centre (de 1000 à 29 999)

Région rurale

Revenu des ménages (référence : quintile de revenu moyen)

Quintile de revenu supérieur

Quintile de revenu moyen-élevé

Quintile de revenu moyen-faible

Quintile de revenu inférieur

Mandataire non de langue anglaise

Religion du mandataire (référence : catholique ou autre religion chrétienne)

Spiritualité autochtone

Agnostique, athée ou areligieux

Bouddhiste

Chrétienne orthodoxe

Hindoue

Juive

Musulmane

Autres

Années des signalements (référence : 2013)

2014

2015

2016

2017

2018

2019

Région sanitaire (référence : Toronto Central) ${ }^{\star}$

Centre

Centre-Est

Centre-Ouest

Champlain

Érié St. Clair

Hamilton Niagara Haldimand Brant

Mississauga Halton

Nord-Est

North Simcoe Muskoka

Nord-Ouest

Sud-Est

Sud-Ouest

Waterloo Wellington

Tercile d'immigration (référence : tercile inférieur)

Tercile moyen de la population d'origine étrangère

Tercile élevé de la population d'origine étrangère
Brut

Ajusté

$0,83(0,80-0,86)$

$0,99(0,98-0,99)$

$1,10(0,99-1,12)$

$0,36(0,29-0,45)$

$1,08(0,96-1,22)$

0,97 (095-099)

$0,72(0,64-0,80)$

$1,37(1,10-1,69)$

$1,09(0,92-1,31)$

$1,87(1,44-2,42)$

$0,86(0,74-1,00)$

$0,91(0,79-1,10)$

$0,97(0,84-1,13)$

$0,92(0,77-1,11)$

$0,97(0,83-1,13)$

$1,11(1,01-1,23)$

$0,27(0,17-0,41)$

$1,64(1,30-2,07)$

$0,31(0,20-0,49)$

$0,27(0,14-0,49)$

$0,53(0,34-0,84)$

$0,35(0,18-0,70)$

$0,14(0,90-0,22)$

$0,58(0,49-0,69)$

$1,07(0,86-1,33)$

$0,91(0,73-1,12)$

$1,16(0,94-1,43)$

$1,23(0,99-1,51)$

$1,21(0,98-1,48)$

$1,16(0,93-1,45)$

$0,53(0,42-0,66)$

$0,78(0,63-0,96)$

$0,86(0,68-1,10)$

$1,12(0,91-1,38)$

$0,93(0,73-1,18)$

$1,13(0,94-1,37)$

$0,67(0,53-0,84)$

$0,67(0,53-0,84)$

$0,80(0,58-1,09)$

$0,58(0,42-0,80)$

$0,79(0,61-1,01)$

$0,81(0,67-0,99)$

$1,02(0,78-1,34)$

$1,00(0,89-1,12)$

$0,74(0,63-0,86)$
$1,06(0,95-1,18)$

$0,46(0,35-0,58)$

$1,01(0,76-1,34)$

$1,00(0,99-1,00)$

$1,03(0,90-1,17)$

$1,26(1,01-1,59)$

$1,12(0,91-1,39)$

$1,73(1,29-2,33)$

$0,80(0,66-0,96)$

$0,81(0,67-0,97)$

$0,88(0,73-1,05)$

$0,89(0,72-1,10)$

$0,87(0,72-1,06)$

1,18(1,02-1,37)

$0,28(0,18-0,46)$

$1,51(1,18-1,93)$

$0,35(0,22-0,56)$

$0,32(0,17-0,62)$

$0,52(0,32-0,85)$

$0,28(0,18-0,81)$

$0,13(0,09-0,23)$

$0,48(0,42-0,54)$

$0,71(0,56-0,92)$

$0,66(0,46-0,94)$

$0,86(0,60-1,23)$

$0,94(0,65-1,35)$

$0,95(0,65-1,39)$

$0,92(0,62-1,36)$

$0,62(0,48-0,80)$

$0,89(0,70-1,13)$

$1,06(0,80-1,41)$

$1,12(0,89-1,42)$

$1,14(0,91-1,42)$

$0,81(0,62-1,05)$

$0,63(0,47-0,84)$

$0,71(0,50-1,01)$

$0,71(0,50-1,01)$

$0,74(0,55-0,99)$

$0,78(0,62-0,98)$

$0,87(0,64-1,19)$

0,93 (0,80-1,09)

$0,80(0,64-0,99)$
$0,89(0,68-1,18)$

Remarque : IC = intervalle de confiance, $\mathrm{RC}=$ rapport de cotes

*Réseaux locaux d'intégration des services de santé en Ontario (RLISS). 
D’autres caractéristiques démographiques des patients ont été associées au consentement. Les patients des petites agglomérations étaient plus susceptibles de consentir que ceux des grands centres urbains (RC 1,73, IC à $95 \%$ 1,29-2,33). Le revenu des ménages a eu un effet variable, indépendamment du quintile. Les patients qui avaient une adresse domiciliaire associée au quintile de revenu plus élevé étaient significativement moins susceptibles de donner leur consentement que les patients du quintile de revenu moyen ( $R C 0,81$, IC à $95 \% 0,67-0,97$ ). Les patients dont l'adresse domiciliaire se situait dans une région présentant le tercile le plus élevé de population née à l'étranger ont présenté la plus faible probabilité de consentement (RC 0,80, IC à 95\% 0,64-0,99).

\section{Interprétation}

Malgré l'augmentation du nombre de donneurs d'année en année, des patients en attente d'une transplantation continuent de décéder, faute d'organes transplantables. Il est crucial d'améliorer l'approche auprès des donneurs potentiels pour appuyer les décisions relatives au consentement, respecter les volontés des patients et abolir les obstacles au don d'organes. Nous avons identifié 3 facteurs potentiellement modifiables indépendamment associés aux approches et au consentement des mandataires, soit : rapidité du signalement aux organisations de don d'organes, consentement téléphonique et participation des médecins à l'approche au consentement.

Les organisations de don d'organes ont besoin de temps pour opérationnaliser l'approche au consentement en personne, particulièrement dans les vastes régions rurales. En conséquence, tout retard à signaler les cas aux organisations de don d'organes aura un effet négatif sur l'approche des donneurs d'organes potentiels. Selon une étude, ces retards ont été la principale cause des dons manqués dans les cas de DCC chez des patients médicalement candidats dont on avait cessé les mesures de maintien des fonctions vitales ${ }^{20}$. Des interventions d'amélioration de la qualité des soins qui mettent l'accent sur la rapidité des signalements ont amélioré les taux de dons aux États-Unis ${ }^{21,22}$, et l'utilisation d'outils décisionnels cliniques électroniques a accéléré les signalements et fait augmenter les dons d'organes dans un hôpital pédiatrique ${ }^{23}$.

Les taux de consentement au don d'organes dépendent du processus d'approche lui-même et de la personne qui en est chargée auprès des mandataires. Même si elles sont rares, nous avons constaté que les approches effectuées par téléphone ont été associées à moins de consentements comparativement aux approches en personne, même après ajustement pour tenir compte d'autres covariables. Les discussions téléphoniques sur le don d'organes limiteraient la capacité des coordonnateurs d'offrir un soutien émotionnel et de répondre au langage non verbal du mandataire; en effet, d'autres études ont révélé que la durée de l'interaction et la qualité du lien créé avec le coordonnateur influent sur les taux de consentements ${ }^{24,25}$. Les organisations de don d'organes pourraient développer des stratégies afin de réduire le recours aux approches téléphoniques.

La mise à contribution d'un coordonnateur dûment formé a été associée à une augmentation des consentements ${ }^{17,26}$. Nous avons observé un taux considérable $(9,2 \%$ des approches réitérées ayant généré 42 donneurs additionnels) lorsqu'un mandataire, qui avait d'abord écarté la possibilité d'un don après mention par l'équipe soignante, a été approché par un coordonnateur dûment formé. Nous avons aussi constaté que les approches au consentement effectuées par des médecins et des coordonnateurs ensemble étaient indépendamment associées à un taux plus élevé de consentements. Même si nous avons procédé à un ajustement pour tenir compte des prémentions du don d'organes par l'équipe soignante, nous ne pouvons pas exclure que ce lien ait subi l'influence d'autres facteurs non mesurés en lien avec le médecin, le mandataire ou la situation clinique. Largement privilégié, le soutien à une approche en collaboration est toutefois mitigé27,28. Le seul essai randomisé ayant évalué les approches en collaboration n'a pas fait état d'une augmentation des taux de consentements ${ }^{28}$, même si cette étude a essuyé des critiques pour sa méthodologie et l'écart entre les groupes étudiés, ce qui limite la confiance à l'endroit de ses conclusions ${ }^{9}$. Cette étude a également comparé les approches impliquant les médecins seulement et les approches en collaboration. Une étude précédente menée au Québec a révélé que la plupart des approches étaient effectuées par des médecins et moins de la moitié impliquaient un coordonnateur ${ }^{29}$. Notre étude est particulière parce que la plupart des cas ont été approchés par un coordonnateur, sans médecin. L'approche collaborative a été appliquée dans moins de $10 \%$ des cas de notre étude, ce qui en fait une piste potentielle d'amélioration ${ }^{28}$. Finalement, nous n'avons observé aucune incidence des médecins responsables des dons dans les hôpitaux sur le consentement. Ces médecins améliorent peut-être les taux de signalements, sans toutefois influer sur les taux de consentements. Nous avons observé une augmentation constante des taux de signalements associée à ces médecins et au cours de la période de l'étude.

Nous avons observé une variabilité géographique pour ce qui est du consentement, même après ajustement pour tenir compte des caractéristiques démographiques des patients, des détails quant à aux approches, et du revenu et de l'ethnicité par région. La variabilité des taux de consentements a déjà été documentée entre les régions administratives ${ }^{25,30}$. Une force de notre étude est la constance du processus attribuable au fait que les dons d'organes sont gérés par une seule organisation. La variabilité des taux de consentements observée pourrait être due au fait que la mentalité et la culture des populations locales face au don d'organes ne sont pas reflétées dans notre étude, ou attribuable à de subtiles nuances régionales quant au processus d'approche.

\section{Limites de l'étude}

Malgré la diversité de notre population, nos conclusions pourraient ne pas être applicables à d'autres régions, car les mentalités face au don d'organes répondent à des facteurs sociaux, démographiques et culturels. La race a un impact sur les taux de dons ${ }^{13,15,31,32}$, et les données personnelles des patients ou des mandataires quant à la race et à l'ethnicité n'étaient pas disponibles pour notre ensemble de données. Nous avons procédé à des ajustements pour le tercile de population née à l'étranger, mais ce marqueur substitut brut de l'ethnicité pourrait ne pas avoir permis un ajustement précis en fonction de l'influence de la race et de la culture sur le consentement. 
Nous avons été incapables de mesurer l'incidence de l'enregistrement des volontés en matière de consentement en raison des politiques de confidentialité en vigueur. Les registres pourraient augmenter les taux de consentements en indiquant aux mandataires les volontés des patients. Selon une étude, plus de la moitié de tous les mandataires ne connaissaient pas les volontés des patients vis-à-vis du don d'organes ${ }^{24,33}$. La création de registres de consentement au don d'organes a été associée à une augmentation de $8 \%$ du taux de dons ${ }^{34}$; la création de registres de don et la sensibilisation du public à leur existence seraient un bon investissement, même si la majorité des personnes inscrites ne deviendront jamais donneurs d'organes ${ }^{35}$. En raison de l'incidence des registres sur les taux de consentement et de la rentabilisation, les gouvernements et les organisations de don d'organes pourraient se concentrer sur des campagnes de mobilisation du public pour promouvoir l'inscription à ces registres.

Nous avons utilisé une imputation multiple pour remplacer les données lacunaires par des valeurs plausibles et calculé les estimations regroupées. La religion des mandataires a été imputée dans $20 \%$ des dossiers, donc le lien avec le consentement devrait être interprété avec prudence. La distribution des différentes confessions religieuses et spirituelles parmi notre cohorte de cas complets était comparable aux données du recensement, ce qui appuie l'hypothèse que ces données ne sont pas aléatoirement lacunaires ${ }^{19}$. Les adresses résidentielles manquantes aux dossiers étaient remplacées par celle de l'établissement de référence, ce qui peut avoir déplacé à tort des patients de régions rurales vers une adresse urbaine où se trouvent les grands hôpitaux et ainsi affaibli la puissance statistique des comparaisons entre les groupes urbains et ruraux. Malgré ces biais potentiels, nous avons observé une différence significative entre les patients des régions urbaines et rurales.

\section{Conclusion}

Le consentement au don d'organes a été associé à des facteurs modifiables dans une grande région multiculturelle dotée d'un système universel de dons d'organes de haut rendement. Des interventions spécifiques visant à assurer la rapidité des signalements aux organisations de don d'organes, à accroître les approches en personne auprès des mandataires en vue d'un consentement et à encourager la participation des médecins au processus d'approche pourraient faire augmenter les taux de consentements au don d'organes.

\section{Références}

1. Views toward organ and tissue donation and transplantation Ottawa: Ipsos Reid [prepared for Canadian Blood Services]; 2010.

2. Organ donation and transplantation in Canada - system progress report 2006-2015. Ottawa: Canadian Blood Services; 2016.

3. Klassen DK, Edwards LB, Stewart DE, et al. The OPTN Deceased Donor Potential Study: implications for policy and practice. Am J Transplant 2016;16:1707-14.

4. Gore SM, Taylor RM, Wallwork J. Availability of transplantable organs from brain stem dead donors in intensive care units. BMJ 1991;302:149-53.

5. Parsons JA. Welsh 2013 deemed consent legislation falls short of expectations. Health Policy 2018;122:941-4.

6. Dominguez J, Rojas JL. Presumed consent legislation failed to improve organ donation in Chile. Transplant Proc 2013;45:1316-7.
7. Beasley CL, Capossela CL, Brigham LE, et al. The impact of a comprehensive, hospitalfocused intervention to increase organ donation. J Transp/ Coord 1997;7:6-13.

8. van Leiden HA, Jansen NE, Haase-Kromwijk BJ, et al. Higher refusal rates for organ donation among older potential donors in the Netherlands: impact of the donor register and relatives. Transplantation 2010;90:677-82.

9. Vincent A, Logan L. Consent for organ donation. Br J Anaesth 2012;108(Suppl 1):i80-7.

10. Guadagnoli E, Christiansen CL, DeJong W, et al. The public's willingness to discuss their preference for organ donation with family members. Clin Transplant 1999;13:342-8.

11. Gortmaker SL, Beasley CL, Sheehy E, et al. Improving the request process to increase family consent for organ donation. J Transpl Coord 1998;8:210-7.

12. Gimbel RW, Strosberg MA, Lehrman SE, et al. Presumed consent and other predictors of cadaveric organ donation in Europe. Prog Transplant 2003;13:17-23.

13. Brown CV, Foulkrod KH, Dworaczyk S, et al. Barriers to obtaining family consent for potential organ donors. J Trauma 2010;68:447-51.

14. Ebadat A, Brown CV, Ali S, et al. Improving organ donation rates by modifying the family approach process. J Trauma Acute Care Surg 2014;76:1473-5.

15. DuBay D, Redden D, Haque A, et al. Is decedent race an independent predictor of organ donor consent or merely a surrogate marker of socioeconomic status? Transplantation 2012;94:873-8.

16. Trillium Gift of Life Network Act, R.S.O. 1990, c. H.20, (1990).

17. Simpkin AL, Robertson LC, Barber VS, et al. Modifiable factors influencing relatives' decision to offer organ donation: systematic review. BMJ 2009;338:b991.

18. Postal Code Conversion File Plus (PCCF+) Version 6A, reference guide. Ottawa: Statistics Canada; 2013.

19. Religion reference guide. In: National Household Survey, 2011. Ottawa: Statistics Canada; 2013:7. Cat no 99-010-X2011010.

20. Krmpotic K, Payne C, Isenor C, et al. Delayed referral results in missed opportunities for organ donation after circulatory death. Crit Care Med 2017;45:989-92.

21. Shafer TJ, Wagner D, Chessare J, et al. US organ donation breakthrough collaborative increases organ donation. Crit Care Nurs Q 2008;31:190-210.

22. Shafer TJ, Wagner D, Chessare J, et al. Organ donation breakthrough collaborative: increasing organ donation through system redesign. Crit Care Nurse 2006;26:33-42, 4-8; quiz 9.

23. Zier JL, Spaulding AB, Finch M, et al. Improved time to notification of impending brain death and increased organ donation using an electronic clinical decision support system. Am J Transplant 2017;17:2186-91.

24. Siminoff LA, Gordon N, Hewlett J, et al. Factors influencing families' consent for donation of solid organs for transplantation. JAMA 2001;286:71-7.

25. Traino HM, Molisani AJ, Siminoff LA. Regional differences in communication process and outcomes of requests for solid organ donation. Am J Transplant 2017; 17:1620-7.

26. Rodrigue JR, Cornell DL, Howard RJ. Organ donation decision: comparison of donor and nondonor families. Am J Transplant 2006;6:190-8.

27. Hulme W, Allen J, Manara AR, et al. Factors influencing the family consent rate for organ donation in the UK. Anaesthesia 2016;71:1053-63.

28. ACRE Trial Collaborators. Effect of "collaborative requesting" on consent rate for organ donation: randomised controlled trial (ACRE trial). BMJ 2009;339:b3911.

29. Baran D, Langevin S, Lebeau C. Organ donation: a portrait of family refusal in Quebec. Transplant Proc 2009;41:3281-3.

30. Goldberg DS, French B, Abt PL, et al. Increasing the number of organ transplants in the United States by optimizing donor authorization rates. Am J Transplant 2015;15:2117-25.

31. Salim A, Berry C, Ley EJ, et al. The impact of race on organ donation rates in Southern California. J Am Coll Surg 2010;211:596-600.

32. Li AH-t, McArthur E, Maclean J, et al. Deceased organ donation registration and familial consent among Chinese and South Asians in Ontario, Canada. PLoS One 2015;10:e0124321.

33. Siminoff LA, Lawrence RH. Knowing patients' preferences about organ donation: does it make a difference? J Trauma 2002;53:754-60.

34. Callison K, Levin A. Donor registries, first-person consent legislation, and the supply of deceased organ donors. J Health Econ 2016;49:70-5.

35. Razdan M, Smith KJ, Bryce CL, et al. Promoting organ donor registries through public education: What is the cost of securing organ donors? Transplantation 2016;100:1332-8. 
Intérêts concurrents : Jeffrey Singh, Michael Hartwick, Karim Soliman, John Boyd, Sonny Dhanani et Andrew Healey reçoivent un salaire du Réseau Trillium pour le don de vie. Ian Ball a reçu un salaire comme médecin responsable des dons d'organes au Réseau Trillium pour le don de vie de 2014 à 2019. John Boyd déclare avoir reçu des subventions de la fondation Physician Services Incorporated, des Instituts de recherche en santé du Canada et de la Société canadienne de soins intensifs, indépendamment des travaux soumis. Il fait partie du Comité de surveillance de la sécurité des données pour l'essai PROACTIVE et a reçu un soutien non financier de la société Edwards LifeSciences pour son programme de recherche, indépendamment des travaux soumis. Aucun autre intérêt n'a été déclaré.

Cet article a été évalué par des pairs.

Affiliations : Division interdépartementale de médecine de soins intensifs (Singh), Université de Toronto; Réseau Trillium pour le don de vie (Singh, Hartwick, Soliman, Boyd, Dhanani, Healey), Toronto, Ont.; Département de médecine et Département d'épidémiologie et de biostatistique (Ball), Université Western, London, Ont.; Département de médecine (Hartwick), Université d'Ottawa, Ottawa, Ont.; Hôpital régional de Windsor (Malus), Windsor, Ont.; École de médecine et de dentisterie Schulich (Malus), London, Ont.; Hôpital Lakeridge Health (Soliman), Oshawa, Ontario, Ont.; Département de médecine de soins intensifs (Boyd), Université Queen's, Kingston, Ont.; Département de pédiatrie (Dhanani), Université d'Ottawa, Ottawa, Ont.; Département de médecine (Healey), Université McMaster, Hamilton, Ont.

Collaborateurs : Tous les auteurs ont largement contribué à la conception et à la modélisation de l'étude, ainsi qu'à l'acquisition, à l'analyse ou à l'interprétation des données. Tous les auteurs ont participé à la rédaction de l'ébauche et aux révisions critiques du manuscrit. Tous les auteurs donnent leur approbation finale pour le manuscrit et assument l'entière responsabilité des travaux, s'étant assurés que les questions ayant trait à l'exactitude ou à l'intégrité de chacun de ses éléments ont été adéquatement étudiées et résolues.

Propriété intellectuelle du contenu : Propriété intellectuelle du contenu : Il s'agit d'un article en libre accès distribué conformément aux modalités de la licence Creative Commons Attribution (CC BY-NC-ND 4.0), qui permet l'utilisation, la diffusion et la reproduction dans tout médium à la condition que la publication originale soit adéquatement citée, que l'utilisation se fasse à des fins non commerciales (c.-à-d., recherche ou éducation) et qu'aucune modification ni adaptation n'y soit apportée. Voir : https://creativecommons.org/licenses/by-nc -nd/4.0/deed.fr.

Financement : Le Réseau Trillium pour le don de vie a fourni une contribution non financière à titre de dépositaire de l'ensemble des données électroniques dont découlent les données utilisées pour cette étude.

Partage des données : L'ensemble des données de cette étude est conservé de manière sécuritaire sous forme encodée et anonymisée au Réseau Trillium pour le don de vie. Les accords de partage des données empêchent les auteurs de rendre public cet ensemble de données. Le plan complet de création, le code d'analyse et les résultats regroupés pour l'ensemble des données sont accessibles auprès des auteurs, sur demande.

Avis : Le Réseau Trillium pour le don de vie n'a pas participé sur le plan de cette étude, ni à l'analyse et au rendu des résultats, ni à l'ébauche du manuscrit et n'y a exercé aucun contrôle ni aucune supervision.

Remerciements : Les auteurs tiennent à remercier le Réseau Trillium pour le don de vie pour son appui à la réalisation de cette étude et pour le soutien administratif et informatique fourni.

Acceptation : Le 23 septembre 2021

Correspondance : Jeffrey Singh, jeff.singh@uhn.ca 